\title{
Вычислительный эксперимент как способ оценки эффективности работы водоподготовительных установок при поточной и противоточной регенерации ионообменных фильтров
}

\author{
Комарова И.В., Галкина Н.К., Прудковский А.Г. \\ Институт геохимии и аналитической химии им. В.И.Вернадского Российской Академии наук, Москва \\ Поступила в редакцию 13.08.2018 г.
}

DOI: https://doi.org/10.17308/sorpchrom.2018.18/606

Проведен вычислительный эксперимент при моделировании процесса очистки воды в условиях поточной и противоточной регенерации, детально изучено влияние способа проведения регенерации на качество и количество очищенной воды на катионите КУ-2. Рассмотрено влияние состава очищаемой воды и глубины очистки воды на выбор схемы регенерации и условий ее проведения.

Ключевые слова: вычислительный эксперимент, поточная и противоточная регенерация, качество и количество очищенной воды

\section{Computer experiment as a method for evaluating the efficiency of water purification in the flow and countercurrent regeneration of ion exchange filters}

\author{
Komarova I.V., Galkina N.K., Prudkovskii A.G. \\ Vernadsky Institute of Geochemistry and Analytical Chemistry of Russian Academy of Sciences. Moscow
}

The aim of this work is to compare co-current and countercurrent regeneration modes in water purification cyclic process on the strong-acid cation exchanger KU-2. Numerical experiments were performed for each cycle which included a sequential calculations for the stages: sorption, regeneration, washing before stabilization at a given amount of reagent for both regeneration modes. Variation of the amount of the regenerative solution was carried out in a wide range

Two types of water, differed from each other in total salt concentration and in the ratio of sodium to the sum of calcium and magnesium, were selected for the comparative study. For each type, water treatment processes were calculated at two values of the degree of purification. The computer experiments were carried out using the program «Createscheme».

Comparison of the efficiency of the regeneration process in the co- and countercurrent flow directions of a regeneration solution was performed on the basis of dependence of the relative amount of purified water, which can be produced on the same bed of the cation exchanger in a hydrogen form, on the amount of the reagent to be used. For both types of water differing in composition and at different requirements to the purification degree, countercurrent regeneration was shown to be more efficient compared to the co-flow mode. Quantitatively, this efficiency depends on the ratio of one - and two-charged ions in raw water and the specified degree of purification. In the entire considered range of changes in the reagent input, the output of purified water of the specified quality is greater at the countercurrent regeneration. The advantage of this regeneration mode is especially pronounced at higher relative content of single-charged sodium ion in fed water and at deeper purification.

Keywords: numerical or computer experiment, co-flow and countercurrent regeneration, quality and quantity of purified water

Комарова и др. / Сорбционные и хроматографические процессы. 2018. Т. 18. № 5 


\section{Введение}

В мировой практике ионообменной водоподготовки начиная с 60-х годов прошлого века наблюдается повышенный интерес к проведению регенерации ионообменных фильтров противоточным методом, поскольку, как известно, выбор способа подачи регенерационного раствора в технологии ионообменной водоподготовки является одним из основных факторов, определяющих эффективность технологии и экономику процесса для действующих и проектируемых схем очистки воды.

Утверждается, что противоточная регенерация существенно рациональнее поточной, приводятся оценки улучшения качества воды, уменьшения затрат, уменьшения расходов реагентов и объемов сточных вод. Одновременно в литературе указаны недостатки противоточной регенерации, в основном касающиеся технической организации процесса и выбора ионитов. Сложность реализации работы фильтров в режиме противоточной регенерации состоит в том, что необходимо во время работы поддерживать слой ионита в зажатом состоянии.

Однако, несмотря на то, что не только ведущие мировые фирмы, такие как Room and Haas, Dow Chemical, Bayer, но и российские специалисты [1-5] усиленно рекомендуют применять в ионообменной водоподготовке противоточную регенерацию, в большинстве технологических схем очистки воды на промышленных установках ТЭС и АЭС в России и других странах все еще используется регенерация поточным методом.

В литературе рассмотрены предложения различных способов организации процесса с противоточной регенерацией: Schewebebett, Amberpack, Upcopre, ВНИАМ и их иодификации, рассматриваются технические приемы организации противоточной регенерации, такие как способ подачи регенерационного раствора, методы зажатия слоя ионита, организация взрыхления ионита или его части. Технологии делятся на две группы в зависимости от направления подачи в фильтр очищаемой воды. Вода поступает сверху вниз при Schwebebett, Upcore. Технология Amberpack предлагает подачу воды снизу вверх, а регенерационного раствора сверху вниз. Общим для всех этих схем является то, что на стадии очистки и регенерации потоки раствора противоположно направлены.

$\mathrm{B}$ настоящей публикации проводится сравнение эффективности поточной и противоточной регенерации на основе рассмотрения динамики ионного обмена в многокомпонентной системе очистки воды на катионите КУ-2 в процессе деминерализации воды путем проведения серии вычислительных экспериментов.

\section{Эксперимент}

В работе использована разработанная нами ранее программа «Createscheme» [6]. Для проведения исследований были выбраны два состава очищаемой воды, соответствующие составам, поступающим на катионит первой ступени в схемах водоподготовки Новочеркасской ГРЭС и Калининской АЭС. Выбор этих вод был обусловлен тем, что ранее мы проводили экспериментальные исследования и расчет процессов очистки воды для этих установок, были изучены особенности промышленного процесса, доказана адекватность математической модели сорбции и регенерации реальному промышленному процессу, проведен расчет отдельных фильтров и схемы в целом $[7,8]$. В обоих случаях регенерация осуществлялась поточным способом. Представлялось целесообразным именно на этих примерах сравнить эффективность поточной и противоточной регенерации. Кроме того эти воды различаются по общему солесодержанию и отношению однозарядного иона натрия к сумме двухза- 
рядных ионов магния и кальция. Режим работы катионита КУ-2 первой ступени очистки влияет на работу последующих фильтров схемы, поэтому был проведен вычислительный эксперимент при разных значениях глубины очистки (табл.1).

Таблица 1. Исходные данные для проведения вычислительного эксперимента

\begin{tabular}{|c|c|c|c|c|}
\hline \multirow[t]{2}{*}{$\begin{array}{c}\text { Станция водоподго- } \\
\text { товки }\end{array}$} & \multirow[t]{2}{*}{$\begin{array}{c}\text { Общее солесодержа- } \\
\text { ние } \mathrm{C}_{0}, \kappa \Gamma-э к в / \mathrm{M}^{3}\end{array}$} & \multirow[t]{2}{*}{$\begin{array}{l}\text { Соотношене } \\
\mathrm{Na} /(\mathrm{Ca}+\mathrm{Mg})\end{array}$} & \multicolumn{2}{|c|}{$\begin{array}{c}\text { Заданная концентрация } \\
\text { натрия в момент проскока, } \\
\text { кг-экв/м }\end{array}$} \\
\hline & & & Вариант 1 & Вариант 2 \\
\hline Калининская АЭС & $3.2310^{-3}$ & 0.17 & $3.4810^{-6}$ & $3.4810^{-4}$ \\
\hline $\begin{array}{c}\text { Новочеркасская } \\
\text { ГРЭС }\end{array}$ & $1.1310^{-2}$ & 0.66 & $1.1310^{-5}$ & $1.1310^{-3}$ \\
\hline
\end{tabular}

Режимные параметры представлены в таблице 2.

Таблица 2. Режимные параметры

\begin{tabular}{|c|c|c|c|c|c|}
\hline \multirow{2}{*}{$\begin{array}{c}\text { Станция } \\
\text { водоподготовки }\end{array}$} & \multirow{2}{*}{$\begin{array}{c}\text { Сечение } \\
\text { фильтра, } \\
\text { м }^{2}\end{array}$} & \multirow{2}{*}{$\begin{array}{c}\text { Высота } \\
\text { слоя ка- } \\
\text { тионита, м }\end{array}$} & \multirow[b]{2}{*}{$\begin{array}{c}\text { Производительность } \\
\text { установки, м/час }\end{array}$} & \multicolumn{2}{|c|}{$\begin{array}{l}\text { Регенерирующй раствор } \\
\left(\mathrm{H}_{2} \mathrm{SO}_{4}\right)\end{array}$} \\
\hline & & & & $\begin{array}{c}\text { Скорость } \\
\text { подачи, } \\
\text { мะ }^{3} / \text { ас }\end{array}$ & $\begin{array}{c}\text { Концентрация } \\
\text { моль/дм }\end{array}$ \\
\hline $\begin{array}{c}\text { Калининская } \\
\text { АЭС }\end{array}$ & 7.06 & 2.0 & 100 & 35 & \multirow{2}{*}{0.179} \\
\hline $\begin{array}{c}\text { Новочеркасская } \\
\text { ГРЭС }\end{array}$ & 7.06 & 2.0 & 60 & 50 & \\
\hline
\end{tabular}

При противоточной регенерации в фильтре отсутствует заполненное водой пространство над слоем ионита и отмывка должна проводиться обессоленной водой [5]. Для корректности сравнительной оценки способа регенерации технологический процесс при поточной и противоточной регенерации рассчитывался при одинаковых условиях: отсутствие водяной подушки и применение частично обессоленной воды для приготовления регенерирующего раствора и отмывки катионита. Расчеты процесса регенерации отвечали использованию разбавленной серной кислоты, как это принято в практике водоподготовки отечественных и зарубежных установок, для того, чтобы избежать выпадения осадка сульфата кальция в катионитовом фильтре.

\section{Обсуждение результатов}

Результаты вычислительного эксперимента представлены ниже в виде выходных кривых очистки и регенерации, распределения катионов по слою катионита после очистки и регенерации, а также в виде зависимости количества очищенной воды заданного качества от продолжительности регенерации.

Первоначально целесообразно было рассмотреть разницу между двумя способами регенерации при одинаковом исходном распределении компонентов по слою ионита. Для этого было выбрано распределение компонентов по слою катионита в результате сорбции на катионите в водородной форме. На рис.1 представлено распределение компонентов по слою катионита в результате очистки воды КАЭС до заданного проскока натрия, равного $3.4810^{-4}$ кг-экв/ $\mathrm{M}^{3}$ на катионите КУ-2 в водородной форме. Это распределение является исходным для расчета и визуализации регенерации потоком и противотоком. 


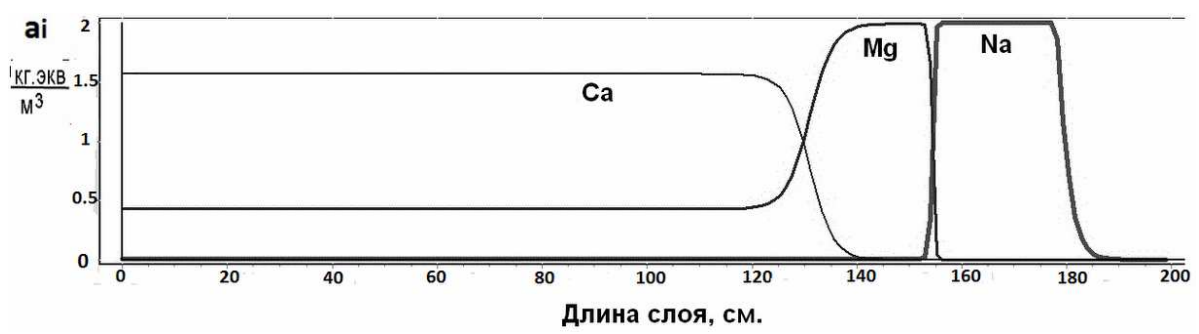

Рис. 1. Распределение компонентов по слою после сорбции на катионите в водородной форме

Наряду с расчетом отдельных стадий программа позволяет осуществлять визуализацию процесса в реальном масштабе времени, заключающуюся в непрерывном наблюдении изменения концентраций всех сорбируемых компонентов на выходе из фильтра и в слое сорбента. Эта информация может быть в дальнейшем использована в управлении процессом для того, чтобы прогнозировать моменты времени, когда необходимо осуществлять изменения режима системы.

В процессе визуализации для выбранного момента времени на выходных кривых регенерации (рис.2) можно получить график распределения всех сорбированных компонетов по слою ионита.

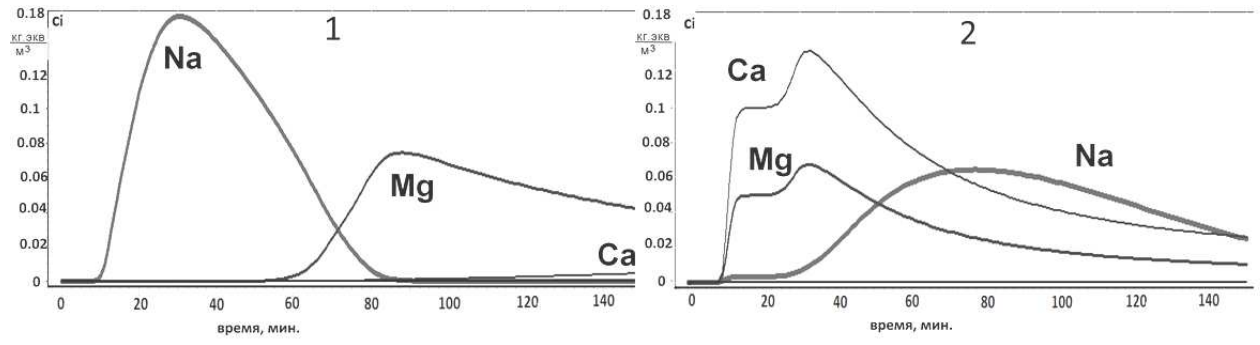

Рис. 2. Выходные кривые регенерации

1-регенерация потоком, 2-регенерация противотоком

На рис. 2 представлены полученные в процессе визуализации для первого технологического цикла выходные кривые регенерации, а на рисунке 3 при трех выбранных моментах времени распределения компонентов по слою ионита при регенерации потоком и противотоком. Эти рисунки наглядно иллюстрируют разницу между двумя способами подачи регенерирующего раствора.

Основным показателем, характеризующим эффективность процесса получения воды, является количество и качество очищенной воды. В данной работе рассмотрена зависимость количества и качества очищенной воды от расхода реагента при двух способах подачи регенерационного раствора. С этой целью проведены расчеты последовательных циклов регенерации катионита заданным расходом кислоты и последующей очистки воды на частично регенерированном катионите до наступления стабилизации. Варьирование времени регенерации (расхода реагента) осуществлялось в широком диапазоне значений.

На рис. 4 представлены результаты расчета одного стабилизированного технологического цикла, состоящего из очистки воды и регенерации катионита для поточного и противоточного способа регенерации. Сравнение потока и противотока приведено при очистке воды КАЭС до проскока натрия. равного $3.4810^{-4}$ кг-экв/ 3 . $\left(\mathrm{C} / \mathrm{C}_{0}=0.1\right)$ при времени регенерации 100 минут. 


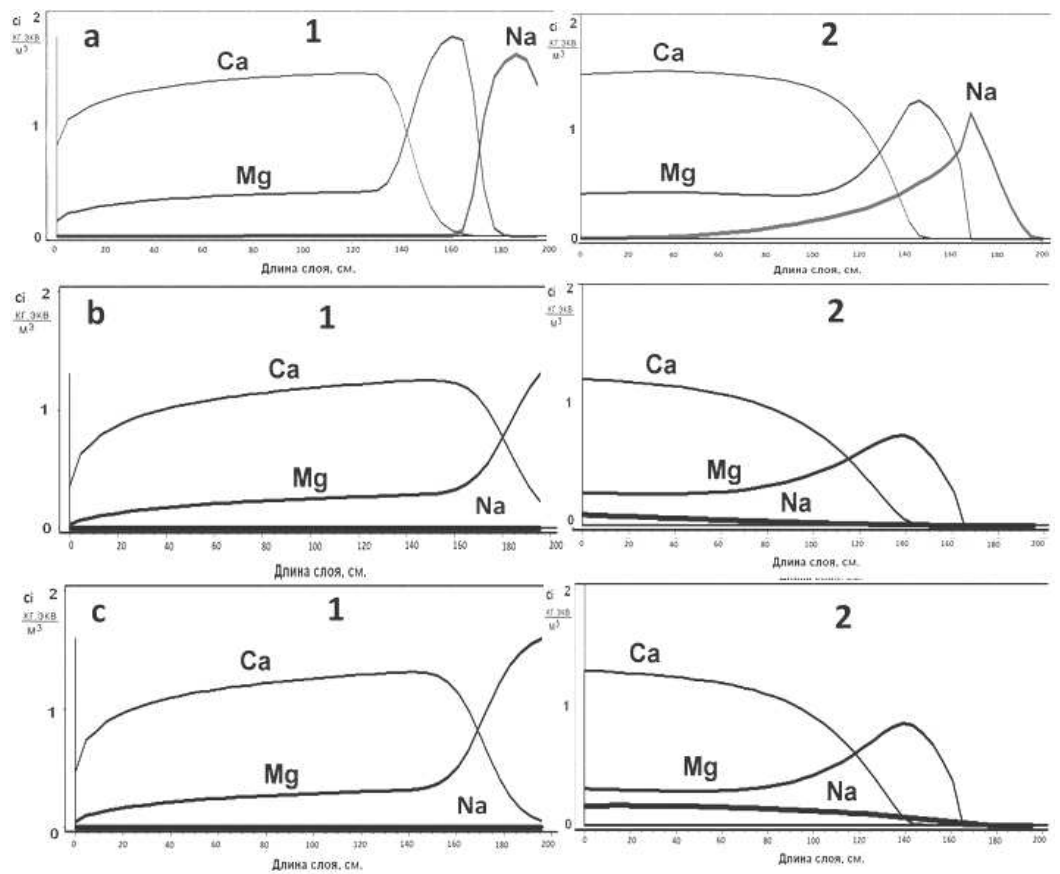

Рис. 3. Распределение компонентов по слою катионита после регенерации в течение a-50 минут, b-100 минут, c- 150 минут. 1- регенерация потоком, 2 - регенерация противотоком
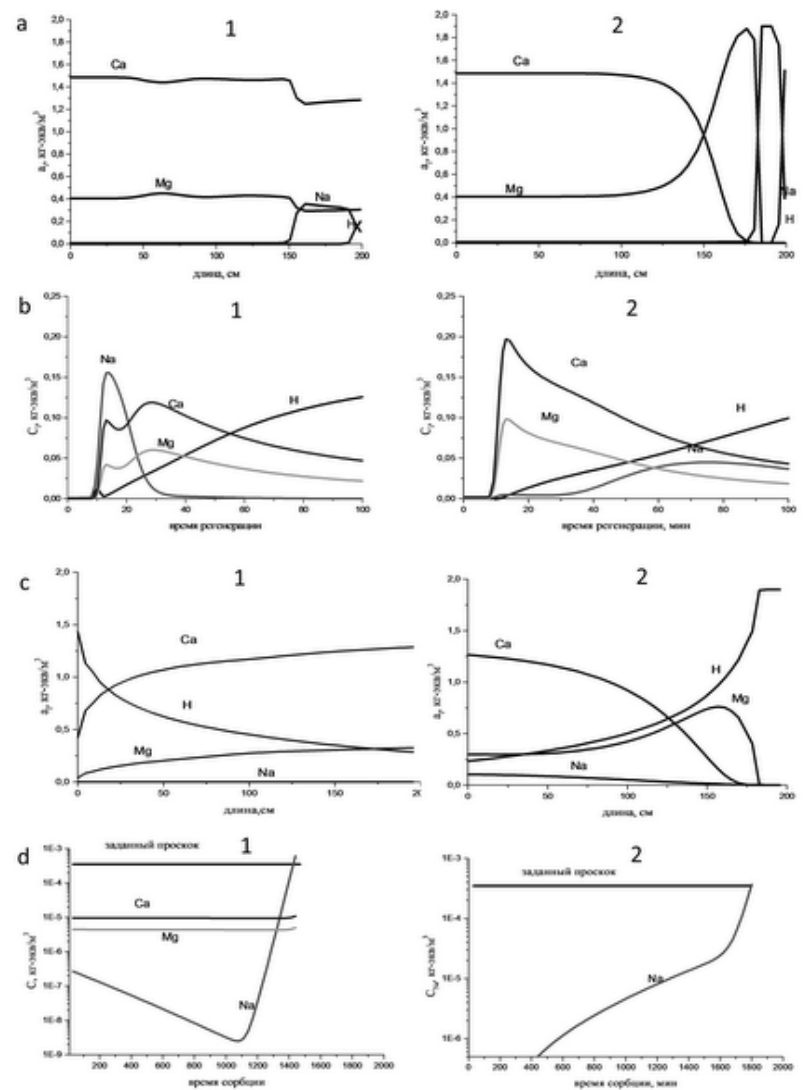

Рис. 4. Результаты расчета стабилизированного технологического цикла очистки воды на катионите КУ-2: регенерация потоком - 1, регенерация противотоком 2. a - распределение компонентов по слою катионита после сорбции; b - выходные кривые регенерации; с -распределение компонентов по слою катионита после регенерации; d - выходные кривые сорбции на частично регенерированном катионите 
Из этих данных следует, что при поточной и противоточной схемах подачи регенерационного раствора распределение компонентов по слою катионита после сорбции и после регенерации, а также выходные кривые регенерации существенно различаются. При поточной регенерации выходная кривая натрия в процессе очистки воды характеризуется двумя участками, первый из которых определяется остаточным содержание иона натрия на выходе из фильтра - концентрация натрия сначала уменьшается за счет вытеснения из замыкающих слоев катионита, а затем нарастает до проскока. Для поточной регенерации характерно также содержание в очищенной воде в небольших количествах ионов кальция и магния.

На рис.5 представлены выходные кривые натрия при сорбции на катионите в водородной форме и на частично регенерированном катионите при стабилизации процесса очистки воды для двух рассматриваемых вод при двух значениях глубины очистки.
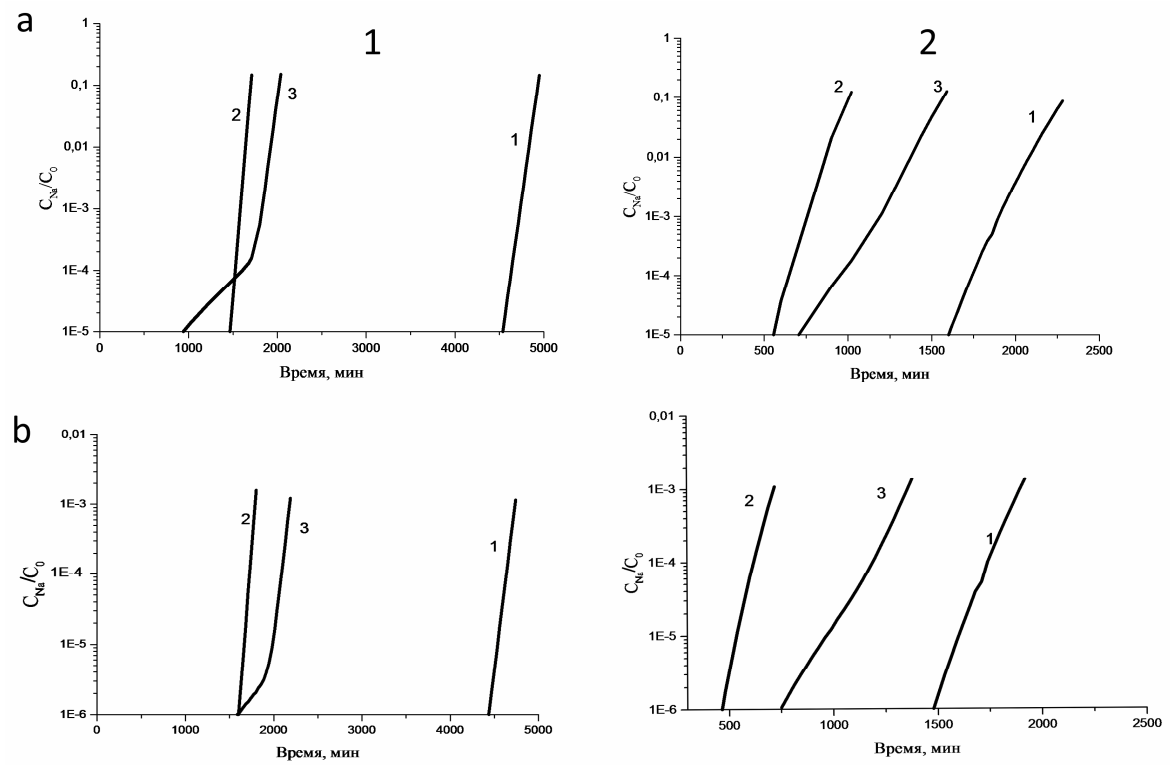

Рис. 5. Выходные кривые натрия: 1 - на катионите КУ-2 в водородной форме; 2 - на частично регенерированном катионите в стабилизированном цикле (регенерация потоком, расход $25 \mathrm{~m}^{3} 0.179 \mathrm{M} \mathrm{H}_{2} \mathrm{SO}_{4}$ ); 3 - на частично регенерированном катионите в стабилизированном цикле (регенерация противотоком, расход $25 \mathrm{~m}^{3}$ $0.179 \mathrm{M} \mathrm{H}_{2} \mathrm{SO}_{4}$ ) при очистке воды КАЭС (1) и воды НГРЭС (2) до проскока натрия $0.1 \mathrm{C}_{0}(\mathrm{a})$ и до проскока натрия $0.001 \mathrm{C}_{0}($ б)

Сравнение способа регенерации проведено по количеству воды, полученной после регенерации, по отношению к количеству воды, очищенной на катионите в водородной форме, при разных расходах кислоты для двух вод разного состава. Полученные данные представлены на рис. 6.

Из этих данных следует, что во всех рассмотренных случаях при очистке двух вод различного состава и разных требованиях к глубине очистки количество воды, очищенной до заданного проскока натрия при противоточной регенерации больше, чем при поточной. Это означает, что противоточная регенерация в целом эффективнее, чем регенерация потоком, однако, степень эффективности в разных случаях разная - она зависит от соотношения одно- и двухзарядных ионов в очищаемой воде и заданной глубины очистки.

Из данных, приведенных далее в таблице 3, следует, что для воды НГРЭС с большим исходным содержанием в этой воде натрия относительное количество очищенной на три порядка воды при противоточной регенерации в 1.8 раза больше, 
чем при регенерации потоком (это справедливо при расходах реагента больше 22 кгмоль, если расходы реагента меньше, глубоко очищенную воду при регенерации потоком получить нельзя из-за вытеснения натрия из замыкающих слоев катионита ионами, поступающими с очищаемой водой).

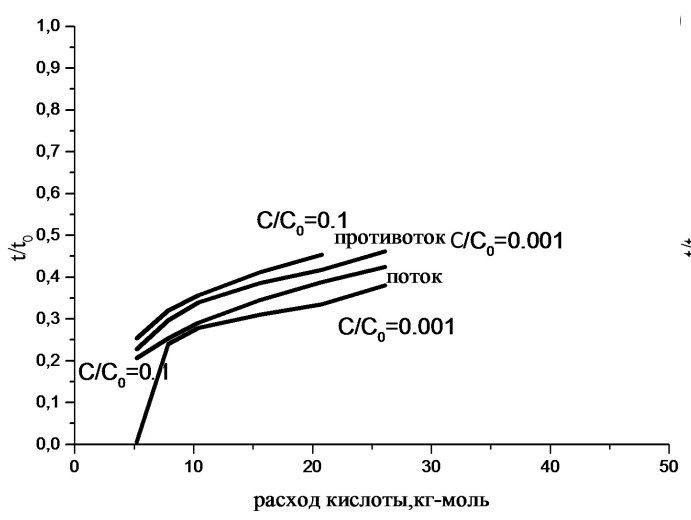

a

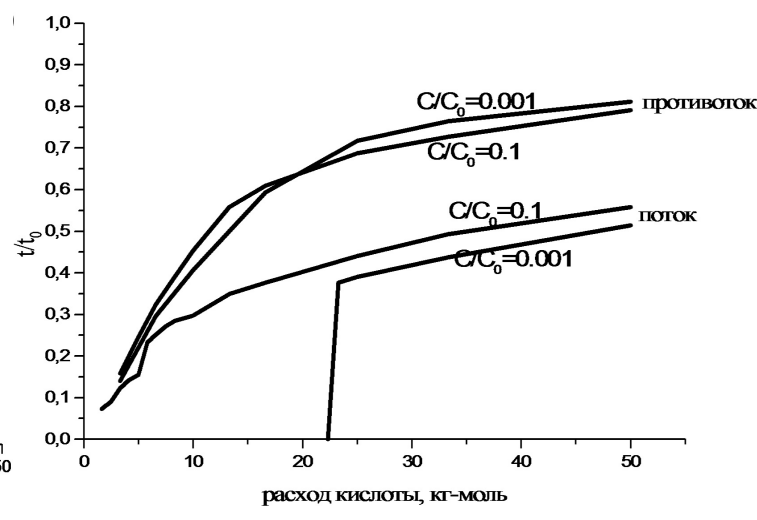

6

Рис. 6. Зависимость относительного количества очищенной воды

(a - КАЭС, б - НГРЭС) от расхода кислоты на регенерацию при двух значениях глубины очистки

Таблица 3. Относительное количество очищенной воды при расходе реагента 25 кг-моль

\begin{tabular}{|c|c|c|c|c|}
\hline \multirow{2}{*}{ Глубина } & \multicolumn{2}{|c|}{ НГРЭС } & \multicolumn{2}{c|}{ КАЭС } \\
\cline { 2 - 5 } & Потоктки & Противоток & Поток & Противоток \\
\hline $\mathrm{C} / \mathrm{C}_{0}=0.1$ & 0.44 & 0.69 & 0.42 & 0.49 \\
\hline $\mathrm{C} / \mathrm{C}_{0}=0.001$ & 0.39 & 0.72 & 0.38 & 0.46 \\
\hline \multicolumn{2}{|c|}{ Соотношение между противотоком и потоком по количеству очищенной воды } \\
\hline $\mathrm{C} / \mathrm{C}_{0}=0.1$ & 1.6 & \multicolumn{2}{c|}{1.2} \\
\hline $\mathrm{C} / \mathrm{C}_{0}=0.001$ & 1.8 & \multicolumn{2}{c|}{1.2} \\
\hline
\end{tabular}

При глубине очистки этой воды на один порядок противоточная регенерация эффективнее в 1.6 раза. Менее заметна разница в количестве получаемой воды в случае очистки воды КАЭС с малым содержанием в ней натрия - при противотоке больше, чем в потоке в 1.2 раза независимо от глубины очистки.

Таким образом, вычислительным экспериментом на примере ионообменной очистки воды на КАЭС и НГРЭС показано, что противоточная регенерация больше всего проявляет свое преимущество перед поточной при глубокой очистке воды с большим относительным содержанием натрия в исходной воде.

\section{Заключение}

Методом вычислительного эксперимента обобщены возможности рационального выбора схемных решений и условий ионообменного технологического процесса регенерации для двух вод, различающихся составом и общим солесодержанием при двух значениях глубины очистки при разных режимах регенерации.

При поточной регенерации имеется минимальный расход реагента, позволяющий получить воду заданного качества. При противоточной регенерации очищенную воду заданного качества можно получить .при любом расходе реагента. Количество очищенной воды заданного качества при противоточной регенерации во всем рассмотренном диапазоне изменения расхода реагента больше, чем при исполь- 
зовании регенерации в потоке. Преимущество противоточной регенерации особенно ярко выражено при большем относительном содержании однозарядного иона натрия в очищаемой воде и при более глубокой очистке.

\section{Список литературы}

1.Громов С.Л. // Теплоэнергетика. 1998. №3. С. 52-55.

2.Боровкова И.И., Балаев И.С., Громов С.Л., Сидоров В.А. и др. // Электрические станциии. 2000. № 5. С. 37-39.

3.Громов С.Л, Пантелеев А.А. // Теплоэнергетика. 2006. № 8. С. 33-37.

4.Юрчевский Г.Ю., Яковлев А.В. // Энергосбережение и водоподготовка. 1998. № 1. C. $52-59$

5.Юрчевский Г.Ю., Комарова И.В., Галкина Н.К., Яковлев А.В. и др. // Теплоэнерге-

тика. 2003. № 7. С. 29-34.

6.Прудковский А.Г., Комарова И.В., Галкина Н.К. // Сорбционные и хроматографические прочессы. 2014. Т. 14. № 5. С.824-831.

7.Комарова И.В., Галкина Н.К., Анфилов Б.Г., Свечинский В.Б. и др. // Наука производству. 1998. № 2(4). С. 53-57.

8.Комарова И.В., Галкина Н.К., Прудковский А.Г., Хамизов Р.Х. // Сорбчионные $u$ хроматографические проиессы. 2017. Т. 17. № 1. C. 31-36.

\section{References:}

1.Gromov S.L., Teplojenergetika, 1998, No 3, pp. 52-55.

2.Borovkova I.I., Balaev I.S., Gromov S.L., Sidorov V.A.et al., Jelektricheskie stancii, 2000, No 5, pp. 37-39.

3.Gromov S.L, Panteleev A.A, Teplojenergetika, 2006, No 8, pp. 33-37.

4.Jurchevskij G.Ju., Jakovlev A.V., Jenergosberezhenie $i$ vodopodgotovka, 1998, No 1, pp. 52-59.

5.Jurchevskij G.Ju., Komarova I.V., Galkina N.K., Jakovlev A.V. et al., Teplojenergetika, 2003, No 7, pp. 29-34.

Комарова Ирина Владимировна - к.Х.н., старший научный сотрудник, лаборатория сорбционных методов, ГЕОХИ РАН, Москва

Галкина Надежда Константиновна - к.х.н., старший научный сотрудник, лаборатория сорбционных методов, ГЕОХИ РАН, Москва

Прудковский Андрей Гаральдович - д.фм.н., старший научный сотрудник, лаборатория сорбционных методов, ГЕОХИ РАН, Москва

6.Prudkovskij A.G., Komarova I.V., Galkina N.K., Sorbtsionnye $i$ khromatograficheskie protsessy, 2014, Vol. 14, No 5, pp. 824-831.

7.Komarova I.V., Galkina N.K., Anfilov B.G., Svechinskij V.B. et al., Nauka proizvodstvu, 1998, No 2(4), pp. 53-57.

8.Komarova I.V., Galkina N.K., Prudkovskij A.G., Hamizov R.H., Sorbtsionnye i $k$ hromatograficheskie protsessy, 2017, Vol. 17, No 1, pp. 31-36.

Komarova Irina V. - Ph.D. (Chem.), Senior researcher, Lab of Sorption Methods, GEOKHI RAS, Moscow

Galkina Nadezda K. - Ph.D. (Chem.), Senior researcher, Lab. of Sorption Methods, GEOKHI RAS, Moscow

Prudkovskii Andrei G. - Dr.Sci. (Phys/Math.), Leading researcher, Lab of Sorption Methods, GEOKHI RAS, Moscow 